\title{
Kinetic Equations for Baryogenesis via Sterile Neutrino Oscillation
}

\author{
Takehiko Asaka ${ }^{1,2}$, Shintaro Eijima ${ }^{2,3}$ and Hiroyuki Ishida ${ }^{2,3}$ \\ ${ }^{1}$ Department of Physics, Niigata University, Niigata 950-2181, Japan \\ ${ }^{2}$ Max-Planck-Institut für Kernphysik, Postfach 103980, 69029 Heidelberg, Germany \\ ${ }^{3}$ Graduate School of Science and Technology, Niigata University, Niigata 950-2181, Japan
}

(December 23, 2011)

\begin{abstract}
We investigate baryogenesis in the $\nu$ MSM (neutrino Minimal Standard Model), which is the MSM extended by three right-handed neutrinos with masses below the electroweak scale. The baryon asymmetry of the universe can be generated by the mechanism via flavor oscillation of right-handed (sterile) neutrinos which are responsible to masses of active neutrinos confirmed by various experiments. We present the kinetic equations for the matrix of densities of leptons which describe the generation of asymmetries. Especially, the momentum dependence of the matrix of densities is taken into account. By solving these equations numerically, it is found that the momentum distribution is significantly distorted from the equilibrium one, since the production for the modes with lower momenta $k \ll T$ ( $T$ is the temperature of the universe) is enhanced, while suppressed for higher modes. As a result, the most important mode for the yields of sterile neutrinos as well as the baryon asymmetry is $k \simeq 2 T$, which is smaller than $\langle k\rangle$ inferred from the thermal average. The comparison with the previous works is also discussed.
\end{abstract}




\section{Introduction}

The baryon asymmetry of the universe (BAU) is one of the most puzzling problems in particle physics and cosmology. It is certain that the Minimal Standard Model (MSM) cannot account for its origin. There have so far been proposed various scenarios of baryogenesis by considering physics beyond the MSM. (See, for example, a recent review [1].) Among them leptogenesis [2] by superheavy right-handed neutrinos is one of the most motivated scenarios. This is because these new particles allow us to give non-zero masses to neutrinos which have been confirmed in many oscillation experiments. Being singlet under the MSM gauge group they can obtain Majorana masses which are completely independent on the electroweak scale. Right-handed neutrinos having superheavy masses then can generate the lepton asymmetry by their decays which can be a source of the BAU. In addition, the observed smallness of active neutrino masses is naturally explained by such fermions through the seesaw mechanism [3]. The required masses are so heavy that it is impossible to directly test these new particles in near future experiments.

Akhmedov, Rubakov and Smirnov have proposed [4] another attractive scenario of baryogenesis by using right-handed neutrinos (mechanism via neutrino oscillation). One of the most important features is that the mechanism works when their masses are smaller than the electroweak scale, which are within the reach of current experiments. The flavor oscillation among right-handed neutrinos in the early universe leads to the separation of the lepton number into right-handed neutrinos and left-handed leptons. The sphaleron process then converts the asymmetry of the left-handed leptons into the baryon asymmetry for high temperatures [5].

This mechanism can be realized in a simple and attractive framework called as the neutrino MSM ( $\nu \mathrm{MSM})$ [6, 7] in which three right-handed neutrinos are introduced with Majorana masses below the electroweak scale. Because of the very suppressed neutrino Yukawa interaction the seesaw mechanism is still effective, which leads to three active neutrinos $\nu_{i}(i=1,2,3)$ and three sterile neutrinos $N_{I}(I=1,2,3)$ as mass eigenstates. The former ones are responsible to the neutrino oscillations observed in experiments. The latter ones give the solutions to the cosmological problems. The lightest sterile neutrino $N_{1}$ with mass $\sim 10 \mathrm{keV}$ can be a candidate of dark matter, while the rest two $N_{2}$ and $N_{3}$ with masses $\sim 1 \mathrm{GeV}$ can generate the BAU through the mechanism above. (For a review see Ref. [8].) Furthermore, the non-minimal coupling of Higgs field to gravity allows to realize the cosmic inflation [9].

Various aspects of baryogenesis in the $\nu$ MSM has been studied until now [7, 10, 11, 12. The generation of the asymmetry is described by the matrix of densities [13, 14, 15] of sterile (right-handed) neutrinos and active (left-handed) leptons. The previous works were based on the kinetic equations for the matrix of densities in Ref. [7], where the terms describing the transfer of asymmetries between sterile neutrinos and active leptons are added to the original ones in [4]. As shown in [7], such terms are essential to generate enough amount of the BAU in the $\nu \mathrm{MSM}$. In spite of their significance such terms are introduced by a heuristic approach.

The main motivation of this article is to improve the estimation of the BAU in the $\nu$ MSM. 
One of the most unsatisfactory points in the previous works is that the evolution of the matrix of densities has been traced only by an approximate way. Namely, the kinetic equations analyzed previously are for the typical, single mode with momentum $k \sim T$ ( $T$ is the temperature of the universe), which is expected to give a dominant contribution to the asymmetry. By extrapolating the result of such a mode to other modes the asymmetry in the number density is estimated.

In this article, thus, we derive the kinetic equations in which the momentum dependence of the matrix of densities is fully taken into account. For this purpose we shall re-evaluate the destruction and production rates of sterile neutrinos as well as active leptons paying attention to the two points. One is the momentum dependence of the particle of interest. The other is the corrections coming from the fact that the states in the destruction and production processes may differ from the thermal equilibrium states (i.e., the deviations from the thermal equilibrium for sterile neutrinos and the chemical potentials for active leptons). As we will show later, the kinetic equations are written as simultaneous integrodifferential equations for the matrices of densities of sterile neutrinos and active leptons.

Interestingly, our kinetic equations include the terms connecting between sterile and active sectors, which is crucial for the baryogenesis in the $\nu \mathrm{MSM}$ as mentioned before. It will be shown that such terms arise automatically as the corrections to the destruction and production rates owing to the deviations from the equilibrium states in the scattering processes. It turns out that the coefficients of these terms are different from [7] and, in addition, there appears a new type of terms which couples sterile neutrinos to active anti-leptons (rather than active leptons).

This paper is organized as follows: In Sec. 2 we briefly review the framework of the analysis and the baryogenesis scenario in the $\nu$ MSM. In the Sec. 3, we derive the kinetic equations which describe the generation of the baryon asymmetry paying attention to the momentum dependence in the matrices of densities. Sec. 4 is devoted to study the numerical solutions to the obtained equations. We show the mode by mode evolution of the matrices of densities and their momentum distributions. The comparison with the previous works is done in Sec. 5. We perform the quantitative comparison of the yields of the BAU, but also clarify the differences between the kinetic equations in the literature. We conclude in Sec. 6.

\section{Baryogenesis in the $\nu \mathrm{MSM}$}

At the beginning we review the $\nu \mathrm{MSM}$, which is the MSM extended by three right-handed neutrinos $\nu_{R I}(I=1,2,3)$ with Lagrangian

$$
\mathcal{L}_{\nu \mathrm{MSM}}=\mathcal{L}_{\mathrm{MSM}}+i \overline{\nu_{R I}} \gamma^{\mu} \partial_{\mu} \nu_{R I}-\left(F_{\alpha I} \bar{L}_{\alpha} \Phi \nu_{R I}+\frac{M_{I}}{2} \overline{\nu_{R I}^{c}} \nu_{R I}+\text { h.c. }\right) .
$$

where $\mathcal{L}_{\mathrm{MSM}}$ is the MSM Lagrangian, $F_{\alpha I}$ are neutrino Yukawa coupling constants, and $\Phi$ and $L_{\alpha}(\alpha=e, \nu, \tau)$ are Higgs and lepton weak-doublets, respectively. The Majorana masses of 
right-handed neutrinos are denoted by $M_{I}$. After the electroweak symmetry breaking neutrinos also obtain the Dirac masses, $\left[M_{D}\right]_{\alpha I}=F_{\alpha I}\langle\Phi\rangle(\langle\Phi\rangle$ is a vacuum expectation value of the Higgs field), and tiny neutrino masses can be explained by the seesaw mechanism if $\left|\left[M_{D}\right]_{\alpha I}\right| \ll M_{I}$ is satisfied. In this case, mass eigenstates of neutrinos are consist of active neutrinos $\nu_{i}(i=1,2,3)$ and sterile neutrinos $N_{I}(I=1,2,3)$. Active neutrinos are the mass eigenstates of ordinary neutrinos and sterile neutrinos are almost the right-handed states $N_{I} \simeq \nu_{R I}$ with masses given by the Majorana masses $M_{I}$ approximately.

On the other hand, when we discuss baryogenesis, the temperatures of interest is higher than the electroweak scale, and we can neglect all the masses of the MSM particles and also the mixing between active and sterile neutrinos. In this case we can treat active and sterile leptons as left-handed leptons and right-handed neutrinos, respectively.

Among three sterile neutrinos, the lightest one $N_{1}$ is a candidate of dark matter. In this dark matter scenario, the coupling constants of neutrino Yukawa interaction of $N_{1}$ are required to be very small. (See, for example, a review [8].) As a result, $N_{1}$ gives the negligible contributions to the seesaw mass matrix of active neutrinos as well as the BAU, and then two sterile neutrinos $N_{2}$ and $N_{3}$ are responsible to these two phenomena.

In the $\nu$ MSM the sufficient amount of baryon asymmetry can be generated [7] by the mechanism via neutrino oscillation [4]. The flavor oscillation between sterile (right-handed) neutrinos $N_{2}$ and $N_{3}$ are induced by the medium effect, which can generate the asymmetries in leptons together with the $\mathrm{CP}$ violation in neutrino Yukawa matrix. The asymmetry in active (left-handed) leptons is then transformed into the baryon asymmetry due to the $B+L$ breaking sphaleron process for high temperatures $T>T_{W}=\mathcal{O}\left(10^{2}\right) \mathrm{GeV}$ [5]. Notice that sterile neutrinos is possible to be out of thermal equilibrium due to the smallness of neutrino Yukawa coupling constants. If this is the case, all the conditions for successful baryogenesis [16] can be satisfied.

In order to describe a series of the processes generating asymmetries, we have to deal with the coherent evolution of sterile neutrinos with the flavor oscillation, and also the incoherent scattering processes with surrounding medium for the destruction and production of sterile neutrinos. We then use the formulation with the matrix of densities [13, 14, 15]. The matrix of interest here is one in the flavor space consisting of sterile neutrinos $N_{2}$ and $N_{3}$ with positive helicities, $\bar{N}_{2}$ and $\bar{N}_{3}$ with negative helicities, active leptons $L_{e}, L_{\mu}$ and $L_{\tau}$, and their antiparticles $\bar{L}_{e}, \bar{L}_{\mu}$ and $\bar{L}_{\tau}$, which leads to a $10 \times 10$ matrix. The diagonal elements of this matrix are nothing but the usual occupation numbers, and the off-diagonal elements contain correlations of the flavor mixings.

In the situation under consideration, however, it can be simplified as described in Ref. [7]. First of all, the conservation of the total lepton number holds for the temperatures of baryogenesis $\left(T>T_{W}\right)$, since Majorana masses $M_{I}$ for sterile neutrinos are sufficiently small in the $\nu$ MSM. We can thus neglect all the elements which break the lepton number. Second, active leptons possess gauge interactions and Yukawa interactions with right-handed charged leptons 
in addition to neutrino Yukawa interactions, which induce large energy gaps between active and sterile states through the thermal effect. Then, the transitions between active and sterile leptons are highly suppressed, and the elements corresponding to the mixing between $L_{\alpha}$ and $N_{I}$ (and also $\bar{L}_{\alpha}$ and $\bar{N}_{I}$ ) are neglected. At this stage, the matrix of densities in the system is decomposed into two $2 \times 2$ matrices $\rho_{N}$ and $\rho_{\bar{N}}$ for $N_{2,3}$ and $\bar{N}_{2,3}$, and two $3 \times 3$ matrices $\rho_{L}$ and $\rho_{\bar{L}}$ for active leptons $L_{e, \mu, \tau}$ and $\bar{L}_{e, \mu, \tau}$. As in Ref. [11], $\rho_{L}$ is considered to be $\rho_{L}=\rho_{\nu}+\rho_{e}=N_{D} \rho_{\nu}$, i.e., the sum of two contributions of lepton doublet. Finally, the off-diagonal elements of $\rho_{L}$ and $\rho_{\bar{L}}$ can be neglected for the temperatures of interest. This is because the flavor transitions among active leptons are also suppressed due to the medium effects induced by the Yukawa interactions of charged leptons with hierarchical coupling constants. Note that active leptons can maintain the kinetic equilibrium due to the rapid interactions with medium, and hence their diagonal matrices of densities can be expressed by using the dimensionless chemical potentials $\mu_{\nu_{\alpha}}$ (the ordinary chemical potential divided by temperature) as

$$
\rho_{L}(k)=N_{D} \rho^{\mathrm{eq}}(k) A, \quad \rho_{\bar{L}}(k)=N_{D} \rho^{\mathrm{eq}}(k) A^{-1},
$$

where $N_{D}=2$ is an $\mathrm{SU}(2)$ factor, $A=\operatorname{diag}\left(e^{\mu_{\nu_{e}}}, e^{\mu_{\nu_{\mu}}}, e^{\mu_{\nu_{\tau}}}\right)$ and $\rho^{\mathrm{eq}}(k)$ is the equilibrium distribution function. Throughout this analysis we apply the approximation of the Boltzmann statistics in which $\rho^{\mathrm{eq}}(k)=e^{-k / T}$.

The evolution of the system is now described by two $2 \times 2$ matrices of densities $\rho_{N}(k)$ and $\rho_{\bar{N}}(k)$, and three differences of the distribution functions $\left[\rho_{L}(k)-\rho_{\bar{L}}(k)\right]_{\alpha \alpha}$ (or three chemical potentials $\left.\mu_{\nu_{\alpha}}\right)$. The previous works on baryogenesis via neutrino oscillations have been based on the kinetic equations for these variables given in Ref. [4] or [7]. The original work [4] has considered the kinetic equations for $\rho_{N}$ and $\rho_{\bar{N}}$ without including the effects from active leptons. As pointed out in Ref. [7, their equations give too small baryon asymmetry to account for the observed value in the $\nu$ MSM. This is because the lightest sterile neutrino $N_{1}$ is a dark matter candidate and essentially only two sterile neutrinos $N_{2}$ and $N_{3}$ participate in baryogenesis. Furthermore, Ref. [7] has introduced the terms which connect active and sterile sectors, and solved the kinetic equations for both $\rho_{N, \bar{N}}$ and $\rho_{L, \bar{L}}$. It has been shown that the additional terms boost the generation of the asymmetry and hence are vital for the successful baryogenesis in the $\nu$ MSM.

There are, however, various aspects to be improved in the previous works towards the precise prediction of the baryon asymmetry in the $\nu$ MSM. One of the most important points is to include correctly the momentum dependence in the matrices of densities $\rho_{N}(k)$ and $\rho_{\bar{N}}(k)$. So far, it has been analyzed only the evolution of the single mode with a typical momentum $k \sim T$, and estimated the asymmetries in number densities by the approximation that the occupation numbers are proportional to the equilibrium one $\rho^{\mathrm{eq}}(k)$. To get rid of this uncertainty, therefore, we will write down the kinetic equations of the matrices of densities for all the modes and solve them numerically taking care of the expansion of the universe. It will be shown later that the momentum distributions of $\rho_{N, \bar{N}}$ are distorted significantly from $\rho^{\mathrm{eq}}$. 


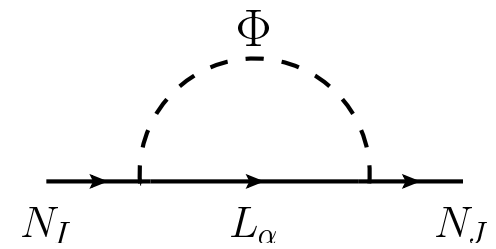

Figure 1: Feynman diagram for self energy of sterile neutrinos.

\section{$3 \quad$ Kinetic Equations}

Now we are at the position to derive the kinetic equations for $\rho_{N, \bar{N}}$ and $\rho_{L, \bar{L}}$. First of all, let us consider the time evolution of $\rho_{N}$. Our construction is based on Ref. [15] (as in [4, 7]) and starts with

$$
\frac{d \rho_{N}\left(k_{N}\right)}{d t}=-i\left[H_{N}\left(k_{N}\right), \rho_{N}\left(k_{N}\right)\right]-\frac{1}{2}\left\{\Gamma_{N}^{d}\left(k_{N}\right), \rho_{N}\left(k_{N}\right)\right\}+\frac{1}{2}\left\{\Gamma_{N}^{p}\left(k_{N}\right), \mathbf{1}-\rho_{N}\left(k_{N}\right)\right\},
$$

where 1 denotes the unit matrix with appropriate dimensions \#1 $H_{N}$ is the effective Hamiltonian, $H_{N}=H_{N}^{0}+V_{N}$, where the free part is $\left[H_{N}^{0}\left(k_{N}\right)\right]_{I J}=E_{N_{I}} \delta_{I J}$ with $E_{N_{I}}=\sqrt{k_{N}^{2}+M_{I}^{2}}$ and $V_{N}$ is the effective potential induced by the medium effects. $\Gamma_{N}^{d}$ and $\Gamma_{N}^{p}$ are the destruction and production rates of $N_{I}$. From now on we shall apply the approximation of the Boltzmann statistics and replace the third term of Eq. (3) as $\frac{1}{2}\left\{\Gamma_{N}^{p}, \mathbf{1}-\rho_{N}\right\} \rightarrow \Gamma_{N}^{p}$.

The first term of Eq. (3) describes the coherent evolution of $\rho_{N}$ and the oscillation of sterile neutrinos occurs due to the off-diagonal elements of $V_{N}$, which is essential for baryogenesis under consideration. It is found from the self energy for sterile neutrinos at finite temperatures in Fig. 1 that the effective potential for the mode $k=k_{N}$ is given by [19]

$$
\left[V_{N}\left(k_{N}\right)\right]_{I J}=\frac{N_{D} T^{2}}{16 k_{N}}\left[F^{\dagger} F\right]_{I J}
$$

where we disregard the correction to $V_{N}$ from the asymmetries in active leptons \#2

In the estimation of $V_{N}$ (as well as $\Gamma_{N}^{d, p}$ below) all masses including $M_{I}$ are neglected since they are irrelevant for temperatures of interest. (Note, however, that we keep $M_{I}$ in $H_{N}^{0}$ because they are crucial for the oscillation of sterile neutrinos.) Further, we first calculate them in the basis where neutrino Yukawa matrix is diagonal, and then find the expression in the original basis shown in Eq. (11).

Let us then estimate the destruction and production rates of $N_{I}$ with momentum $k_{N}$. In the considering temperatures the dominant contributions come from the scattering processes (A) $N_{I}+Q_{L} \leftrightarrow L_{\alpha}+t_{R}$, (B) $N_{I}+\bar{t}_{R} \leftrightarrow L_{\alpha}+\bar{Q}_{L}$, and (C) $N_{I}+\bar{L}_{\alpha} \leftrightarrow t_{R}+\bar{Q}_{L}$ [4], shown in

\footnotetext{
\#1 We have neglected the non-linear effects of $\rho_{N}$ since the interaction rates between sterile neutrinos are sufficiently small. Otherwise, see Ref. [17, 18.

\#2 We have numerically confirmed that the change of the final baryon asymmetry by this effect is negligibly small.
} 

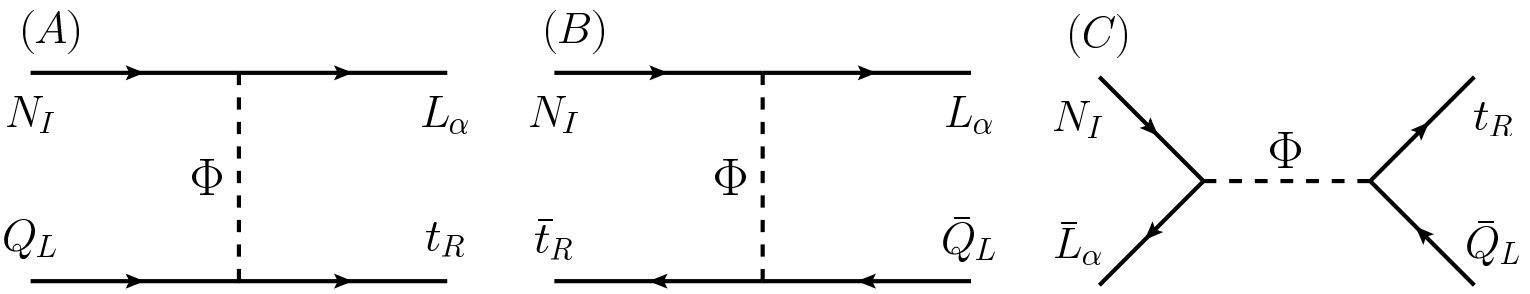

Figure 2: Feynman diagrams for scattering processes of production and destruction rates.

Fig. 2. Here $Q_{L}$ and $t_{R}$ denote left-handed quark doublet of third generation and right-handed top quark. We then divide the rates into three parts:

$$
\Gamma_{N}^{d, p}\left(k_{N}\right)=\Gamma_{N}^{d, p(\mathrm{~A})}\left(k_{N}\right)+\Gamma_{N}^{d, p(\mathrm{~B})}\left(k_{N}\right)+\Gamma_{N}^{d, p(\mathrm{C})}\left(k_{N}\right)
$$

The destruction rates of each process are found to be

$$
\begin{aligned}
& {\left[\Gamma_{N}^{d(\mathrm{~A})}\left(k_{N}\right)\right]_{I J}=\left[\Gamma_{N}^{d(\mathrm{~B})}\left(k_{N}\right)\right]_{I J}=\gamma_{N}^{d}\left(k_{N}\right)\left[F^{\dagger} F\right]_{I J}} \\
& {\left[\Gamma_{N}^{d(\mathrm{C})}\left(k_{N}\right)\right]_{I J}=\gamma_{N}^{d}\left(k_{N}\right)\left[F^{\dagger} F\right]_{I J}+\left[\delta \Gamma_{N}^{d}\left(k_{N}\right)\right]_{I J}}
\end{aligned}
$$

Here we have introduced

$$
\gamma_{N}^{d}\left(k_{N}\right)=\frac{N_{C} N_{D} h_{t}^{2}}{64 \pi^{3}} \frac{T^{2}}{k_{N}},
$$

where $N_{C}=3$ is a color factor and $h_{t} \simeq 1$ is the top Yukawa coupling constant, and

$$
\begin{aligned}
{\left[\delta \Gamma_{N}^{d}\left(k_{N}\right)\right]_{I J} } & =\gamma_{N}^{d}\left(k_{N}\right) \int_{0}^{\infty} \frac{d k_{L} k_{L}}{N_{D} T^{2}}\left[F^{\dagger}\left(\rho_{\bar{L}}^{T}\left(k_{L}\right)-N_{D} \rho^{\mathrm{eq}}\left(k_{L}\right) \mathbf{1}\right) F\right]_{I J} \\
& =\gamma_{N}^{d}\left(k_{N}\right)\left[F^{\dagger}\left(A^{-1}-\mathbf{1}\right) F\right]_{I J}
\end{aligned}
$$

where we have used Eq. (2) in the last equality. On the other hand, the production rates are

$$
\begin{aligned}
& {\left[\Gamma_{N}^{p(\mathrm{~A})}\left(k_{N}\right)\right]_{I J}=\left[\Gamma_{N}^{p(\mathrm{~B})}\left(k_{N}\right)\right]_{I J}=\gamma_{N}^{d}\left(k_{N}\right) \rho^{\mathrm{eq}}\left(k_{N}\right)\left[F^{\dagger} F\right]_{I J}+\left[\delta \Gamma_{N}^{p}\left(k_{N}\right)\right]_{I J},} \\
& {\left[\Gamma_{N}^{p(\mathrm{C})}\left(k_{N}\right)\right]_{I J}=\gamma_{N}^{d}\left(k_{N}\right) \rho^{\mathrm{eq}}\left(k_{N}\right)\left[F^{\dagger} F\right]_{I J},}
\end{aligned}
$$

where

$$
\begin{aligned}
{\left[\delta \Gamma_{N}^{p}\left(k_{N}\right)\right]_{I J}=\gamma_{N}^{d}\left(k_{N}\right) \rho^{\mathrm{eq}}\left(k_{N}\right) } & \left\{\int_{0}^{k_{N}} \frac{d k_{L}}{N_{D} k_{N}} \frac{1-\rho^{\mathrm{eq}}\left(k_{L}\right)}{\rho^{\mathrm{eq}}\left(k_{L}\right)}\left[F^{\dagger}\left(\rho_{L}\left(k_{L}\right)-N_{D} \rho^{\mathrm{eq}}\left(k_{L}\right) \mathbf{1}\right) F\right]_{I J}\right. \\
& \left.+\int_{k_{N}}^{\infty} \frac{d k_{L}}{N_{D} k_{N}} \frac{1-\rho^{\mathrm{eq}}\left(k_{N}\right)}{\rho^{\mathrm{eq}}\left(k_{N}\right)}\left[F^{\dagger}\left(\rho_{L}\left(k_{L}\right)-N_{D} \rho^{\mathrm{eq}}\left(k_{L}\right) \mathbf{1}\right) F\right]_{I J}\right\}^{\mathrm{eq}}\left(k_{N}\right)\left[F^{\dagger}(A-\mathbf{1}) F\right]_{I J}
\end{aligned}
$$


It should be noted that $\delta \Gamma_{N}^{d}$ and $\delta \Gamma_{N}^{p}$ vanish when all the chemical potentials of active leptons become zero (i.e., $\left.\rho_{L}(k)=\rho_{\bar{L}}(k)=N_{D} \rho^{\mathrm{eq}}(k) \mathbf{1}\right)$. In this case, the rates of each process satisfy $\Gamma_{N}^{p(\mathrm{~A}, \mathrm{~B}, \mathrm{C})}\left(k_{N}\right)=\rho^{\mathrm{eq}}\left(k_{N}\right) \Gamma_{N}^{d(\mathrm{~A}, \mathrm{~B}, \mathrm{C})}\left(k_{N}\right)$, and the total rates are given by

$$
\left[\Gamma_{N}^{d \mathrm{eq}}\left(k_{N}\right)\right]_{I J}=3 \gamma_{N}^{d}\left(k_{N}\right)\left[F^{\dagger} F\right]_{I J}, \quad\left[\Gamma_{N}^{p \mathrm{eq}}\left(k_{N}\right)\right]=\rho^{\mathrm{eq}}\left(k_{N}\right)\left[\Gamma_{N}^{d \mathrm{eq}}\left(k_{N}\right)\right]_{I J} .
$$

The kinetic equations for $\rho_{N}$ and $\rho_{\bar{N}}$ are then summarized as

$$
\begin{aligned}
\frac{d \rho_{N}\left(k_{N}\right)}{d t}= & -i\left[H_{N}^{0}\left(k_{N}\right)+V_{N}\left(k_{N}\right), \rho_{N}\left(k_{N}\right)\right]-\frac{1}{2}\left\{\Gamma_{N}^{d \mathrm{eq}}\left(k_{N}\right), \rho_{N}\left(k_{N}\right)-\rho^{\mathrm{eq}}\left(k_{N}\right) 1\right\} \\
& +2 \delta \Gamma_{N}^{p}\left(k_{N}\right)-\frac{1}{2}\left\{\delta \Gamma_{N}^{d}\left(k_{N}\right), \rho_{N}\left(k_{N}\right)\right\} .
\end{aligned}
$$

The equation for $\rho_{\bar{N}}$ can be found by the CP conjugation, i.e., by exchanging $F \leftrightarrow F^{*}$ and $\rho_{L} \leftrightarrow \rho_{\bar{L}}\left(\right.$ or $\left.A \leftrightarrow A^{-1}\right)$.

Next, we turn to consider the left-handed leptons. Similar to the above case, we start with the equation for $\rho_{L}$ as

$$
\frac{d \rho_{L}\left(k_{L}\right)}{d t}=-i\left[H_{\nu}\left(k_{L}\right), \rho_{L}\left(k_{L}\right)\right]-\frac{1}{2}\left\{\Gamma_{\nu}^{d}\left(k_{L}\right), \rho_{L}\left(k_{L}\right)\right\}+N_{D} \Gamma_{\nu}^{p}\left(k_{L}\right) .
$$

Here remember that $\rho_{L}$ denotes the sum of $\mathrm{SU}(2)$ doublet contributions $\rho_{L}=\rho_{\nu}+\rho_{e}$. As explained in Eq. (2), only the diagonal elements of both sides are taken into account and then the first term of right-hand side becomes irrelevant for the discussion. Further, active leptons maintain the kinetic equilibrium as Eq. (2) due to the large interaction rates, we have only to consider the evolution of their chemical potentials.

$$
\cosh \mu_{\nu_{\alpha}} \frac{d \mu_{\nu_{\alpha}}}{d t}=\frac{1}{4 N_{D}} \int \frac{d k_{L} k_{L}^{2}}{T^{3}} \frac{d}{d t}\left[\rho_{L}\left(k_{L}\right)-\rho_{\bar{L}}\left(k_{L}\right)\right]_{\alpha \alpha} .
$$

We then estimate the destruction and production rates of active (left-handed) neutrinos, $\Gamma_{\nu}^{d}$ and $\Gamma_{\nu}^{p}$, caused by neutrino Yukawa interaction. Similar to sterile neutrinos, the dominant contributions are found from the scattering processes $(\mathrm{A}),(\mathrm{B})$ and $(\mathrm{C})$. The production rates are then estimated as

$$
\begin{aligned}
& {\left[\Gamma_{\nu}^{d(\mathrm{~A})}\left(k_{L}\right)\right]_{\alpha \beta}=\left[\Gamma_{\nu}^{d(\mathrm{~B})}\left(k_{L}\right)\right]_{\alpha \beta}=\gamma_{\nu}^{d}\left(k_{L}\right)\left[F F^{\dagger}\right]_{\alpha \beta},} \\
& {\left[\Gamma_{\nu}^{d(\mathrm{C})}\left(k_{L}\right)\right]_{\alpha \beta}=\gamma_{\nu}^{d}\left(k_{L}\right)\left[F F^{\dagger}\right]_{\alpha \beta}+\left[\delta \Gamma_{\nu}^{d}\left(k_{L}\right)\right]_{\alpha \beta},}
\end{aligned}
$$

where

$$
\begin{aligned}
\gamma_{\nu}^{d}\left(k_{L}\right) & =\frac{N_{C} h_{t}^{2} T^{2}}{64 \pi^{3} k}=\frac{1}{N_{D}} \gamma_{N}^{d}\left(k_{L}\right) \\
{\left[\delta \Gamma_{\nu}^{d}\left(k_{L}\right)\right]_{\alpha \beta} } & =\gamma_{\nu}^{d}\left(k_{L}\right) \int_{0}^{\infty} \frac{d k_{N} k_{N}}{T^{2}}\left[F\left(\rho_{\bar{N}}^{T}\left(k_{N}\right)-\rho^{\mathrm{eq}}\left(k_{N}\right) \mathbf{1}\right) F^{\dagger}\right]_{\alpha \beta} .
\end{aligned}
$$

On the other hand, the production rates for three processes are

$$
\begin{aligned}
& {\left[\Gamma_{\nu}^{p(\mathrm{~A})}\left(k_{L}\right)\right]_{\alpha \beta}=\left[\Gamma_{\nu}^{p(\mathrm{~B})}\left(k_{L}\right)\right]_{\alpha \beta}=\gamma_{\nu}^{d}\left(k_{L}\right) \rho^{\mathrm{eq}}\left(k_{L}\right)\left[F F^{\dagger}\right]_{\alpha \beta}+\left[\delta \Gamma_{\nu}^{p}\left(k_{L}\right)\right]_{\alpha \beta},} \\
& {\left[\Gamma_{\nu}^{p(\mathrm{C})}\left(k_{L}\right)\right]_{\alpha \beta}=\gamma_{\nu}^{d}\left(k_{L}\right) \rho^{\mathrm{eq}}\left(k_{L}\right)\left[F F^{\dagger}\right]_{\alpha \beta},}
\end{aligned}
$$


where

$$
\begin{aligned}
{\left[\delta \Gamma_{\nu}^{p}\left(k_{L}\right)\right]_{\alpha \beta}=\gamma_{\nu}^{d}\left(k_{L}\right) \rho^{\mathrm{eq}}\left(k_{L}\right) } & \left\{\int_{0}^{k_{L}} \frac{d k_{N}}{k_{L}} \frac{1-\rho^{\mathrm{eq}}\left(k_{N}\right)}{\rho^{\mathrm{eq}}\left(k_{N}\right)}\left[F\left(\rho_{N}\left(k_{N}\right)-\rho^{\mathrm{eq}}\left(k_{N}\right) \mathbf{1}\right) F^{\dagger}\right]_{\alpha \beta}\right. \\
+ & \left.\int_{k_{L}}^{\infty} \frac{d k_{N}}{k_{L}} \frac{1-\rho^{\mathrm{eq}}\left(k_{L}\right)}{\rho^{\mathrm{eq}}\left(k_{L}\right)}\left[F\left(\rho_{N}\left(k_{N}\right)-\rho^{\mathrm{eq}}\left(k_{N}\right) \mathbf{1}\right) F^{\dagger}\right]_{\alpha \beta}\right\} .
\end{aligned}
$$

The rates of left-handed charged leptons are the same as those of active neutrinos, and the $\mathrm{CP}$ conjugation $\left(F \leftrightarrow F^{*}\right.$ and $\left.\rho_{N} \leftrightarrow \rho_{\bar{N}}\right)$ gives the rates for $\bar{\nu}$. The equations for chemical potentials are then given by

$$
\begin{aligned}
& \frac{d \mu_{\nu_{\alpha}}}{d t}=-\gamma_{\nu}^{d}(T)\left[F F^{\dagger}\right]_{\alpha \alpha} \tanh \mu_{\nu_{\alpha}} \\
&+\frac{\gamma_{\nu}^{d}(T)}{4} \int_{0}^{\infty} \frac{d k_{N} k_{N}}{T^{2}}\left\{\left(1+\frac{2}{\cosh \mu_{\nu_{\alpha}}}\right)\left[F \rho_{N}\left(k_{N}\right) F^{\dagger}-F^{*} \rho_{\bar{N}}\left(k_{N}\right) F^{T}\right]_{\alpha \alpha}\right. \\
& \\
&\left.-\tanh \mu_{\nu_{\alpha}}\left[F \rho_{N}\left(k_{N}\right) F^{\dagger}+F^{*} \rho_{\bar{N}}\left(k_{N}\right) F^{T}\right]_{\alpha \alpha}\right\} .
\end{aligned}
$$

Therefore, the three equations, (12) for $\rho_{N}$, the CP conjugation of (12) for $\rho_{\bar{N}}$, and (20) for $\mu_{\nu_{\alpha}}$, are the kinetic equations for our study of baryogenesis in the $\nu \mathrm{MSM}$.

Here are some comments. First, we have fully taken into account the momentum dependence in the matrices of densities $\rho_{N}$ and $\rho_{\bar{N}}$. As a result, the kinetic equations are written as simultaneous integrodifferential equations, which allow us to find the distribution of the occupation numbers of sterile neutrinos, the significant modes to generate the baryon asymmetry, and the temperatures crucial for baryogenesis.

Second, we have calculated the destruction and production rates of sterile neutrinos and active leptons for a given momentum. It should be noted that we have taken into account the case when the initial and final states in scattering processes which are not in the thermal equilibrium and estimate the corrections to the rates from the deviations $\rho_{N, \bar{N}}-\rho^{\text {eq }}$ and $\rho_{L, \bar{L}}-$ $N_{D} \rho^{\text {eq }}$. Interestingly, the communication terms between $\rho_{N}$ and $\rho_{L}\left(\rho_{\bar{N}}\right.$ and $\left.\rho_{\bar{L}}\right)$ introduced in Ref. [7] arise automatically, which is the term with $\delta \Gamma_{N}^{p}$ in Eq. (12). Now we can understand well the origin of such terms. For instance, $\delta \Gamma_{N}^{p}$ originates in the correction to the production rate of $N$ in the scattering processes (A) and (B) when chemical potentials of active leptons are non-zero. The similar discussion can be applied to the communication terms in Eq. (20). As shown in Ref. [7, they play a crucial role to generate the BAU in the $\nu$ MSM.

Finally, our kinetic equations also contain the communication terms between $\rho_{N}$ and $\rho_{\bar{L}}\left(\rho_{\bar{N}}\right.$ and $\rho_{L}$ ) which have not been discussed before. For example, the term with $\delta \Gamma_{N}^{d}$ in Eq. (12) corresponds to it. Such a term arises as the corrections to the destruction rates of sterile neutrinos due to the non-zero chemical potentials of active leptons via the scattering process (C). The important point is that the term with $\delta \Gamma_{N}^{d}$ is the second order of the matrices of 
densities (i.e., $\rho_{N} \rho_{\bar{L}}$ ) and the kinetic equations are no longer linear in $\rho_{N}, \rho_{\bar{N}}$ and $\mu_{\nu_{\alpha}}$. Further, the presence of these terms is essential to ensure the conservation of the lepton number.

The total lepton number becomes a conserved charge for temperatures of baryogenesis since the Majorana masses are much smaller than $T_{W}$, as mentioned before. In the considering system, this leads to

$$
0=\frac{d}{d t}\left\{R^{3}\left[\sum_{I=2,3}\left(n_{N_{I}}-n_{\bar{N}_{I}}\right)+\sum_{\alpha=e, \mu, \tau}\left(n_{L_{\alpha}}-n_{\bar{L}_{\alpha}}\right)\right]\right\},
$$

where $R$ is the scale factor of the expanding universe and $n_{X}$ is the number density of particle $X$. It can be written in terms of the matrices of densities (and chemical potentials) as

$$
\begin{aligned}
0 & =\int \frac{d^{3} k}{(2 \pi)^{3}} \frac{d}{d t} \operatorname{tr}\left[\rho_{N}(k)-\rho_{\bar{N}}(k)+\rho_{L}(k)-\rho_{\bar{L}}(k)\right] \\
& =\int \frac{d^{3} k}{(2 \pi)^{3}} \frac{d}{d t} \operatorname{tr}\left[\rho_{N}(k)-\rho_{\bar{N}}(k)\right]+\frac{2 N_{D} T^{3}}{\pi^{2}} \cosh \mu_{\nu_{\alpha}} \frac{d \mu_{\nu_{\alpha}}}{d t}
\end{aligned}
$$

A simple calculation shows that our equations (12) and (20) satisfy this equality. It is found for the scattering processes (A) and (B) that the production (or destruction) terms in $\rho_{N}$ and $\rho_{\bar{N}}$ cancel with the destruction (or production) terms in $\rho_{L}$ and $\rho_{\bar{L}}$, respectively, after taking the trace and integrating over momentum. As for the process $(\mathrm{C})$ the production (or destruction) terms in $\rho_{N}$ and $\rho_{\bar{N}}$ cancel with the production (or destruction) terms in $\rho_{\bar{L}}$ and $\rho_{L}$, respectively. Thus, if the system starts with the lepton symmetric universe, the asymmetry of sterile neutrinos is always opposite to that of active leptons. Namely, the kinetic equations here describe not the generation of the total lepton asymmetry (i.e., leptogenesis), but the separation into sterile and active sectors.

\section{Numerical Solution of Kinetic Equations}

In this section, we shall study the numerical solution of the kinetic equations (12) and (20). To incorporate the expansion of the universe we replace the time derivative in Eq. (12) as $\frac{d}{d t} \rightarrow \frac{\partial}{\partial t}-H k \frac{\partial}{\partial k}$, where $H=T^{2} / M_{0}\left(M_{0}=7.12 \times 10^{17} \mathrm{GeV}\right)$ is the Hubble expansion rate. The time-temperature relation is then given by $\frac{d T}{d t}=\frac{T^{3}}{M_{0}}$. In this analysis, we take the initial conditions as $\rho_{N}(k)=\rho_{\bar{N}}(k)=\mu_{\nu_{\alpha}}=0$, which may be realized by the primordial inflation. We solve these equations till $T=T_{W}$ in order to estimate the BAU, where $T_{W}$ is the sphaleron freezing temperature. (See, for example Ref. [21].) Following to Ref. [12], we set $T_{W}=140$ GeV from now on.

In solving the kinetic equations, we have to specify neutrino Yukawa coupling constants $F_{\alpha I}$ and masses of sterile neutrinos $M_{2}$ and $M_{3}$. The estimation of the baryon asymmetry in full parameter region is beyond the scope of this analysis, which is postponed in another publication. Here we choose a specific choice of parameters and study the properties of the solutions. 

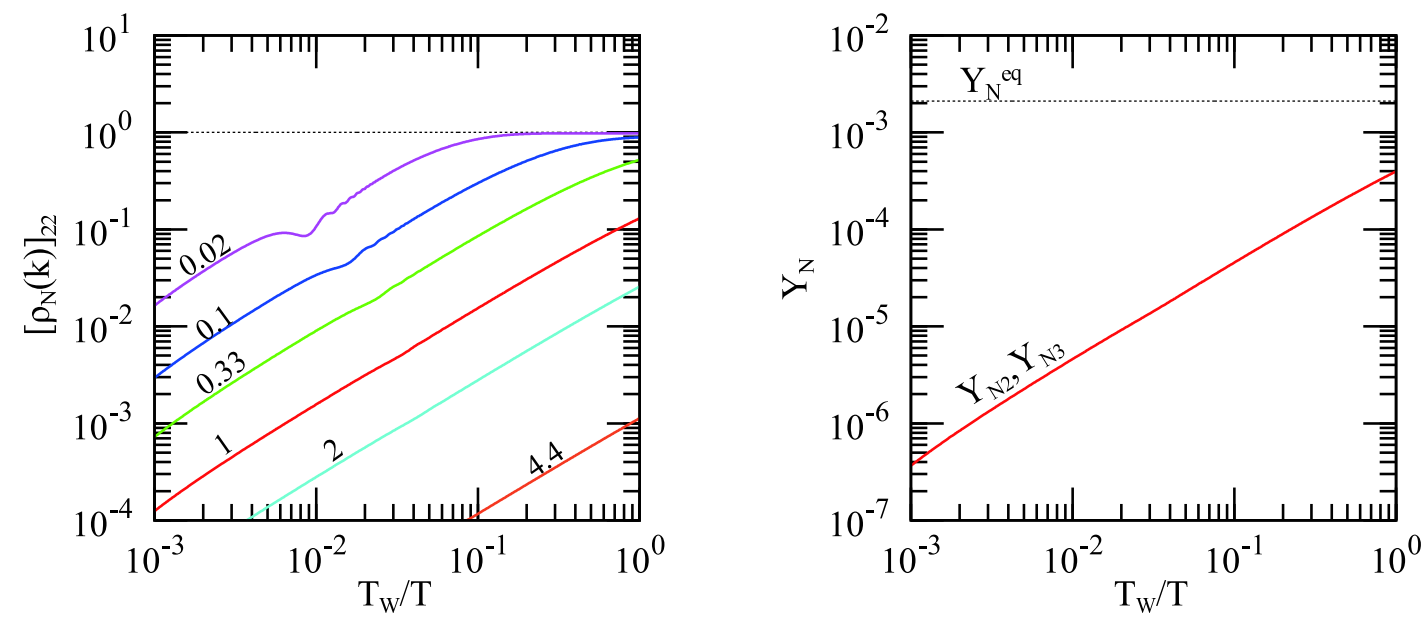

Figure 3: Evolution of $\left[\rho_{N}(k)\right]_{22}$ with $k / T=0.02,0.1,0.33,1$, 2 and 4.4 in the left panel, and evolution of $Y_{N_{2,3}}$ in the right panel.

As for the neutrino Yukawa couplings we follow the notation in Ref. [22]. In this analysis we consider the normal hierarchy of active neutrino masses and take $m_{3}=4.89 \times 10^{-2} \mathrm{eV}$, $m_{2}=8.71 \times 10^{-3} \mathrm{eV}, m_{1}=0, \sin ^{2} \theta_{23}=0.42, \sin ^{2} \theta_{12}=0.312$ and $\sin ^{2} \theta_{13}=0.025$ [20]. Further, we choose the parameters of sterile neutrinos as $\xi=+1, \omega=\pi / 4, \delta=7 \pi / 4$ and $\eta=\pi / 3$. We write masses of sterile neutrinos as $M_{3}=M_{N}+\Delta M_{N} / 2$ and $M_{2}=M_{N}-\Delta M_{N} / 2$, and choose, as representative values, $M_{N}=10 \mathrm{GeV}$ by fixing $\Delta M_{N} / M_{N}=10^{-8}$ otherwise stated.

The diagonal elements of the matrices of densities $\rho_{N}$ and $\rho_{\bar{N}}$ are the occupation numbers of $N_{I}$ and $\bar{N}_{I}$. The evolution of these quantities are shown in the left panel of Fig. 3. In the present choice of parameters (i.e., $\omega=\pi / 4$ ) the occupation numbers of $N_{2}$ and $N_{3}$ are almost the same, and we only show the evolution of $\left[\rho_{N}\right]_{22}$. It is seen that the occupation numbers increase as the temperature decreases due to the production by the scattering processes (A), (B) and (C). In Eq. (12) the third term on the right-hand side which proportional to $\rho^{\text {eq }}$ is the main source of the production of sterile neutrinos. Since the destruction rate in Eq. (111) is inversely proportional to the momentum, the production of modes with lower momenta is more effective. We find that the low modes stop to grow eventually and take constant values given by $\left[\rho_{N}(k)\right]_{I I}=\left[\rho_{\bar{N}}(k)\right]_{I I}=\rho^{\mathrm{eq}}(k)$ afterward. This thermalization temperature becomes higher for the mode with smaller momentum.

The yields of $N_{I}$ and $\bar{N}_{I}$, defined by the ratio between the number and entropy densities, are given by

$$
Y_{N_{I}}=\frac{1}{s} \int \frac{d^{3} k}{(2 \pi)^{3}}\left[\rho_{N}(k)\right]_{I I}, \quad Y_{\bar{N}_{I}}=\frac{1}{s} \int \frac{d^{3} k}{(2 \pi)^{3}}\left[\rho_{\bar{N}}(k)\right]_{I I},
$$

where the entropy density is $s=\frac{2 \pi^{2}}{45} g_{s} T^{3}$ with $g_{s}=106.75$. When the system is fully thermalized (i.e., $\left.\left[\rho_{N}(k)\right]=\left[\rho_{\bar{N}}(k)\right]=\rho^{\mathrm{eq}}(k) \mathbf{1}\right)$, the yields take a constant value

$$
Y_{N_{I}}^{\mathrm{eq}}=Y_{\bar{N}_{I}}^{\mathrm{eq}}=\frac{45}{2 g_{s} \pi^{4}}=2.1 \times 10^{-3},
$$



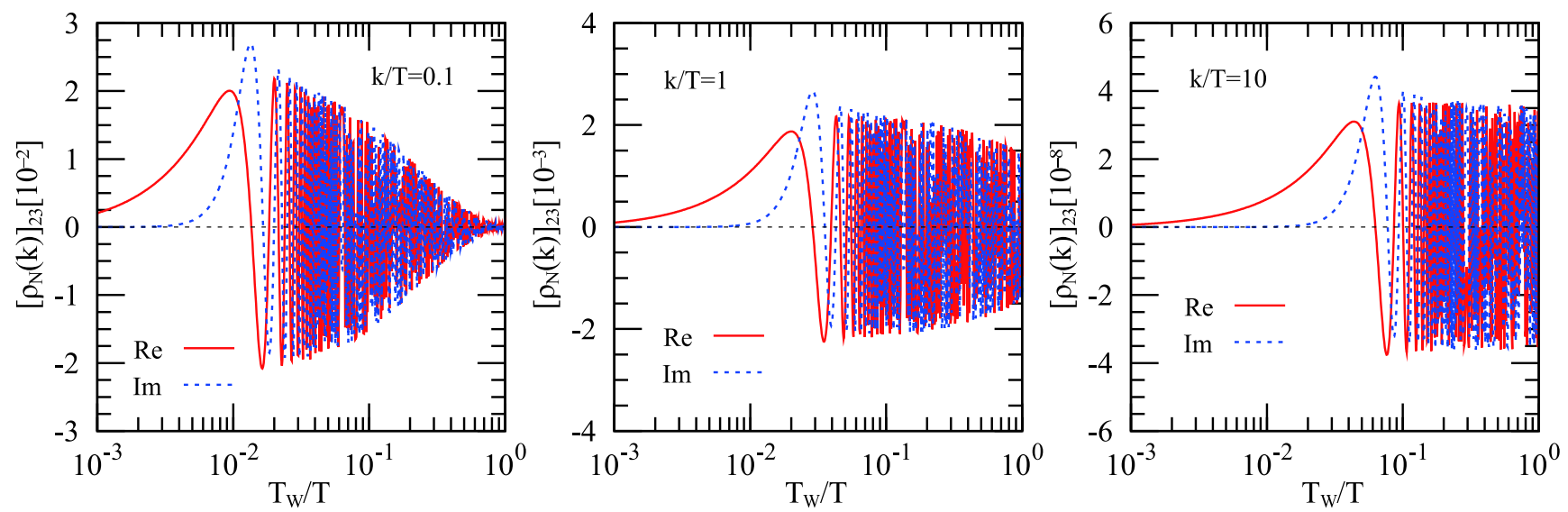

Figure 4: Evolution of $\operatorname{Re}\left[\rho_{N}\right]_{23}$ (red solid lines) and $\operatorname{Im}\left[\rho_{N}\right]_{23}$ (blue dashed lines) for the modes with $k / T=0.1$ (left panel), 1 (middle panel) and 10 (right panel), respectively.

for the temperatures of interest. It is found that $Y_{N_{2}}$ and $Y_{N_{3}}$ increase as $1 / T$ until they close to the equilibrium values as shown in Fig. 3 .

On the other hand, the off-diagonal elements of the matrices of densities contain correlations of the flavor mixing. In Fig. 4 we show the evolution of $\left[\rho_{N}(k)\right]_{23}$ for the modes with $k / T=0.1$, 1 and 10. It is seen that both real and imaginary parts of $\left[\rho_{N}(k)\right]_{23}$ start to oscillate around the temperature [7]

$$
T_{\mathrm{osc}}(k)=\left(\frac{M_{0} \Delta M_{N} M_{N}}{3(k / T)}\right)^{1 / 3},
$$

and hence the oscillation for the mode with lower momentum begins at higher temperature. We should note that the amplitude of the oscillation becomes damped and the off-diagonal elements of $\rho_{N}$ vanish when the mode gets in the thermal equilibrium. This behavior can be seen for the mode with $k / T=0.1$ in Fig. 4 .

The flavor oscillation between $N_{2}$ and $N_{3}$ can generate the asymmetries of sterile neutrinos as well as active leptons [4, 7], together with the $\mathrm{CP}$ violation in neutrino sector. The analytical description of these production processes are found in Refs. [4, 17, 10, 11], and we just show the results of the numerical analysis.

The asymmetries in the occupation numbers of sterile neutrinos, $\left[\Delta \rho_{N}(k)\right]_{I I}=\left[\rho_{N}(k)\right]_{I I}-$ $\left[\rho_{\bar{N}}(k)\right]_{I I}$, are generated as shown in the left panel of Fig. 5. It is seen that such an asymmetry for the mode with $k$ is generated at $T \simeq T_{\text {osc }}(k)$ and the absolute value of the asymmetry becomes larger for the lower modes. We also find that the asymmetries of the mode with $k / T=0.02$ begins to damp at $T_{W} / T \simeq 0.2$, at which the mode gets in thermal equilibrium. Having the asymmetries of each mode, we can estimate the asymmetry in the number density as

$$
Y_{\Delta N_{I}}=\frac{1}{s} \int \frac{d^{3} k}{(2 \pi)^{3}}\left[\Delta \rho_{N}(k)\right]_{I I} .
$$



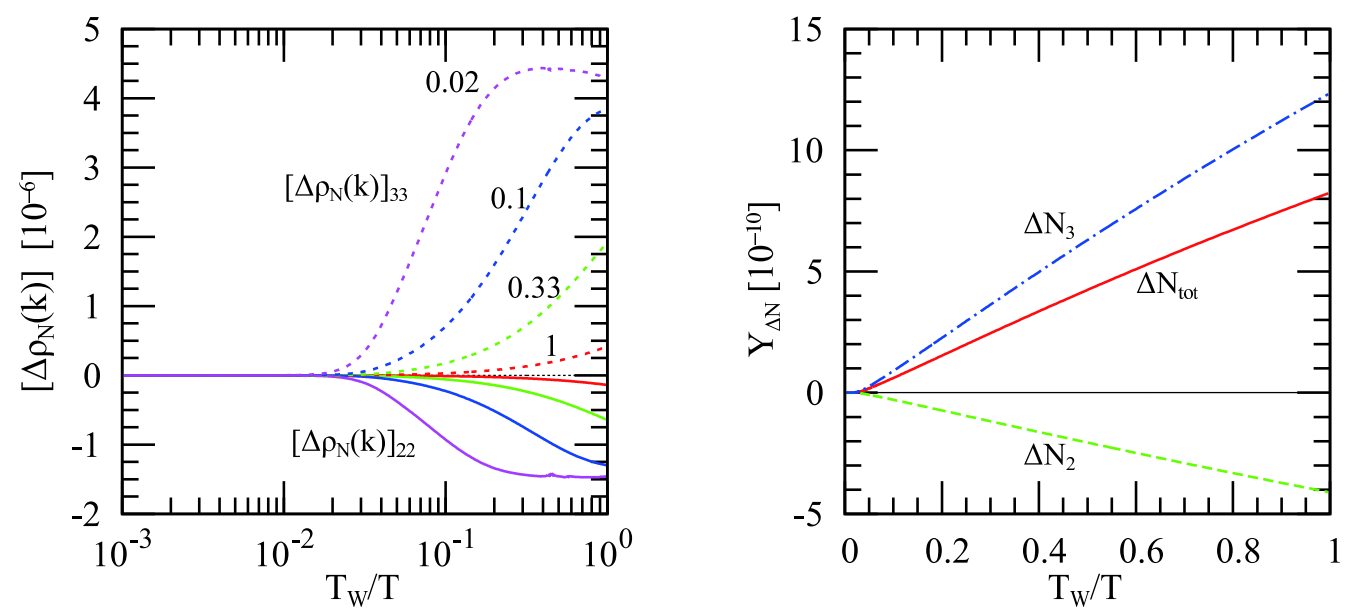

Figure 5: Evolution of $\left[\Delta \rho_{N}\right]_{22}$ (solid lines) and $\left[\Delta \rho_{N}\right]_{33}$ (dotted lines) with momenta $k / T=$ 0.02, 0.1, 0.33 and 1 in the left panel, and evolution of $Y_{\Delta N_{2}}, Y_{\Delta N_{3}}$ and $Y_{\Delta N_{\text {tot }}}$ in the right panel.
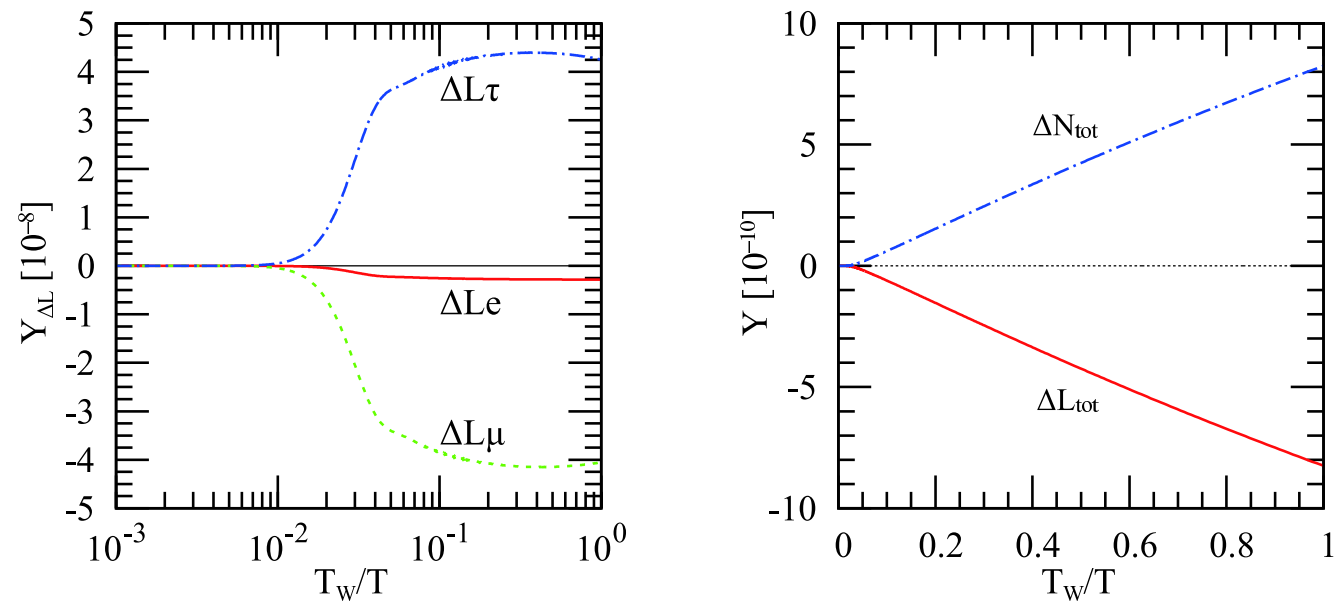

Figure 6: Evolution of $Y_{\Delta L_{e}}, Y_{\Delta L_{\mu}}$ and $Y_{\Delta L_{\tau}}$ in the left panel, and evolution of $Y_{\Delta L_{\mathrm{tot}}}$ and $Y_{\Delta N_{\mathrm{tot}}}$ in the right panel.

As shown in the right panel of Fig. 5, $Y_{\Delta N_{I}}$ increases as $\sim 1 / T$ as long as the system is away from the equilibrium state. We also show the evolution of total asymmetry of sterile neutrinos, $Y_{\Delta N_{\text {tot }}}=Y_{\Delta N_{2}}+Y_{\Delta N_{3}}$.

In the considering system, the non-zero asymmetry in the sterile sector necessarily indicates the presence of asymmetry in active sector. The yields of active flavor asymmetries are expressed in terms of chemical potentials as

$$
Y_{\Delta L_{\alpha}}=\frac{45 N_{D}}{g_{s} \pi^{4}} \sinh \mu_{\alpha}
$$

The evolution of $Y_{\Delta L_{\alpha}}$ is shown in the left panel of Fig. 6. The asymmetries of active flavors $Y_{\Delta L_{\alpha}}$ are larger than those of sterile neutrinos $Y_{\Delta N_{I}}$. This is because $Y_{\Delta L_{\alpha}}$ is induced at the fourth order of neutrino Yukawa coupling constants, while $Y_{\Delta N_{I}}$ is at the sixth order [7]. As shown in 
Ref. [11] when $\operatorname{Im} \omega=0$, the yield $Y_{\Delta L_{\alpha}}$ is proportional to the CP asymmetry parameter $A_{32}^{\alpha}$. In the present choice of parameters, they are

$$
A_{32}^{e}: A_{32}^{\mu}: A_{32}^{\tau}=-1.0:-15: 16
$$

It is then found that our numerical solutions of the kinetic equations reproduce the analytic relations of asymmetries of active lepton flavors.

The total asymmetry stored in active leptons is now obtained as $Y_{\Delta L_{\mathrm{tot}}}=Y_{\Delta L_{e}}+Y_{\Delta L_{\mu}}+$ $Y_{\Delta L_{\tau}}$, which evolution is shown in the right panel of Fig. 6. For comparison we also show the asymmetry in sterile sector. It is clearly seen that $Y_{\Delta N_{\text {tot }}}+Y_{\Delta L_{\text {tot }}}=0$ due to the conservation of the total lepton number in the considering system. These total asymmetries are obtained as the $\mathcal{O}\left(F^{6}\right)$ effects. We also find that the generation of the total asymmetries becomes effective at the temperature

$$
T_{L} \sim T_{\mathrm{osc}}(k=T)
$$

We find $T_{L} \sim 6.2 \times 10^{3} \mathrm{GeV}$ for our choice of parameters. These yields scale as $1 / T$, which are consistent with the estimation in Ref. [7], even if one includes the momentum dependence of the matrices of densities properly.

The asymmetry in active sector can be partially converted into the baryon asymmetry as $\Delta B=-\frac{28}{79} \Delta L_{\text {tot }}$ due to the rapid sphaleron transition [5]. The BAU observed in the present universe is then given by

$$
Y_{B}=-\left.\frac{28}{79} Y_{\Delta L_{\mathrm{tot}}}\right|_{T=T_{W}}=-1.53 \times\left. 10^{-3} N_{D} \sum_{\alpha} \sinh \mu_{\nu_{\alpha}}\right|_{T=T_{W}} .
$$

Notice that the asymmetry for $T<T_{W}$ cannot contribute to the BAU since the sphaleron process is ineffective for the lower temperatures. In the considering parameter choice, the BAU is given by (See also the case III in Tab. 1.)

$$
Y_{B}=\left\{\begin{array}{ll}
2.9 \times 10^{-10} & \text { for } M_{N}=10 \mathrm{GeV} \\
5.4 \times 10^{-14} & \text { for } M_{N}=100 \mathrm{MeV}
\end{array} .\right.
$$

When the sphaleron process is frozen at $T=T_{W}$, the distribution of the occupation number $\left[\rho_{N}(k)\right]_{22} \simeq\left[\rho_{N}(k)\right]_{33}$ is represented in terms of momentum in the left panel of Fig. 7 . We should stress here that the momentum dependence in the occupation number $\left[\rho_{N}(k)\right]_{I I}$ is significantly different from the equilibrium one $\rho^{\mathrm{eq}}(k)$. This is because of the momentum dependence in the destruction and production rates of sterile neutrinos estimated in the previous section. These rates are characterized by $\gamma_{N}^{d}(k)$ in Eq. (7) and $\gamma_{N}^{d}(k) \propto 1 / k$. This results in the enhancement and the suppression of the production of the mode with lower and higher momentum than about $T$, respectively. Especially, the low modes with $k / T \lesssim 0.1$ are equilibrated until $T=T_{W}$ and coincides with $\rho^{\mathrm{eq}}(k)$. It is also found that the most significant mode for the number density is found to be $k \simeq 2 T$, which is smaller than $\langle k\rangle=3 T$ obtained by the thermal average. 

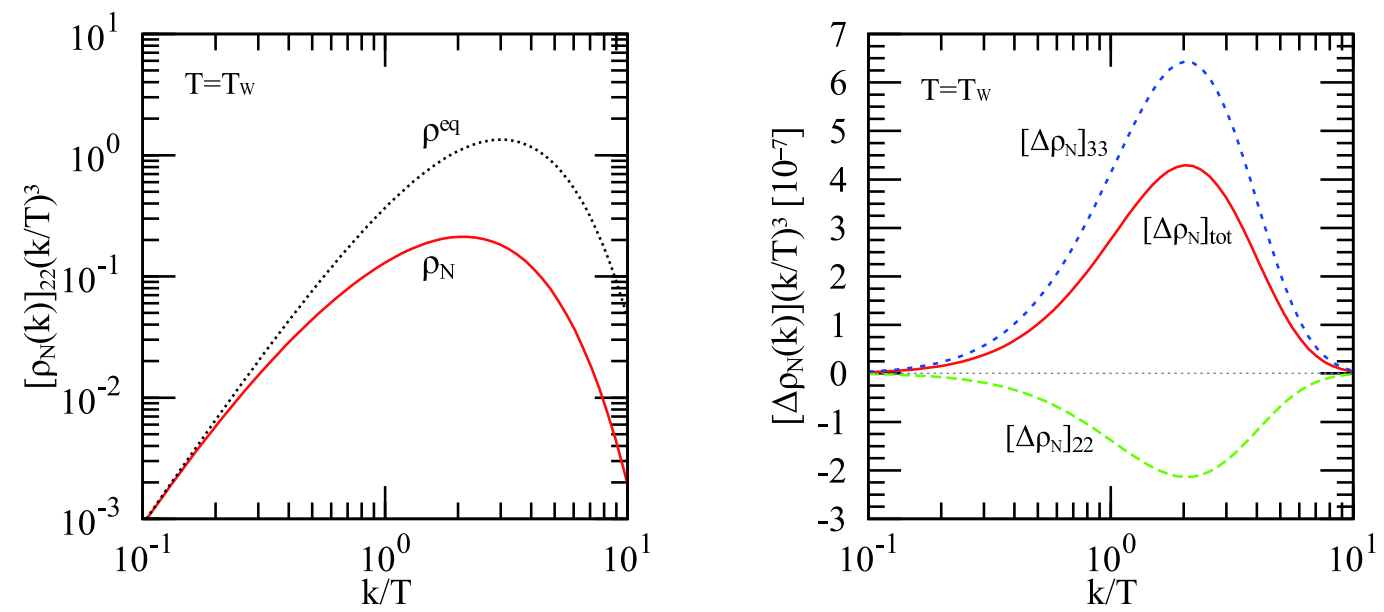

Figure 7: Momentum distributions of $\left[\rho_{N}(k)\right]_{22}$ (red solid line) and $\rho^{\mathrm{eq}}(k)$ (black dotted line) in the left panel. Momentum distributions of $\left[\Delta \rho_{N}(k)\right]_{22}$ (green dashed line), $\left[\Delta \rho_{N}(k)\right]_{33}$ (blue dotted line), and $\left[\Delta \rho_{N}(k)\right]_{\text {tot }}$ (red solid line) in the right panel. The distributions are evaluated at $T=T_{W}$ and are multiplied by $(k / T)^{3}$.

In the right panel of Fig. 7 we show the momentum distributions of the asymmetries of the occupation numbers, $\left[\Delta \rho_{N}(k)\right]_{22},\left[\Delta \rho_{N}(k)\right]_{33}$ and their sum $\left[\Delta \rho_{N}(k)\right]_{\text {tot }}$, at $T=T_{W}$. Similar to the occupation numbers, they have different momentum distributions from $\rho^{\mathrm{eq}}(k)$. (See Fig. 8.) Interestingly, the most important mode for the total asymmetry in sterile sector (and hence for the BAU) is found to be $k \simeq 2 T$ as before.

Therefore, these characteristic behaviors of the distributions show that the momentum dependence in the matrices of densities (as well as the destruction and production rates) is indispensable for the correct evaluation of the BAU in the $\nu \mathrm{MSM}$.

\section{Comparison with previous works}

In this section we shall compare the results obtained in this analysis with the previous works, especially given in Ref. [11], and also estimate the qualitative difference on the BAU. So far the momentum dependence of $\rho_{N}$ and $\rho_{\bar{N}}$ have been approximately taken into account. To make the differences clear, let us first consider what kind of approximations should be imposed on the kinetic equations (12) and (20) in order to reproduce the previous results.

It is then found that there are three important assumptions. (i) The momentum dependence is approximated by the equilibrium one, namely,

$$
\left[\rho_{N}(k)\right]_{I J}=\left[R_{N}\right]_{I J} \rho^{\mathrm{eq}}(k), \quad\left[\rho_{\bar{N}}(k)\right]_{I J}=\left[R_{\bar{N}}\right]_{I J} \rho^{\mathrm{eq}}(k),
$$

and the evolution of $R_{N}$ and $R_{\bar{N}}$ is studied. (ii) The thermal average is taken for the destruction rates of sterile neutrinos. (iii) The destruction and production rates by the scattering process (C) are identified with those by the process (A) or (B). We will discuss these points in turn. 
First of all, based on the assumption (i), the equations for $R_{N}$ and chemical potentials are from Eqs. (12) and (201) as

$$
\begin{aligned}
\frac{d R_{N}}{d t}= & -i\left[H_{N}(k), R_{N}\right]-\frac{3 \gamma_{N}^{d}(k)}{2}\left\{F^{\dagger} F, R_{N}-\mathbf{1}\right\}+2 \gamma_{N}^{d}(k) F^{\dagger}(A-\mathbf{1}) F \\
& -\frac{\gamma_{N}^{d}(k)}{2}\left\{F^{\dagger}\left(A^{-1}-\mathbf{1}\right) F, R_{N}\right\} \\
\frac{d \mu_{\nu_{\alpha}}}{d t}= & -\frac{3 \gamma_{\nu}^{d}(T)}{2}\left[F F^{\dagger}\right]_{\alpha \alpha} \tanh \mu_{\alpha}+\frac{\gamma_{\nu}^{d}(T)}{2}\left[F R_{N} F^{\dagger}-F^{*} R_{\bar{N}} F^{T}\right]_{\alpha \alpha} \frac{1}{\cosh \mu_{\alpha}} \\
& +\frac{\gamma_{\nu}^{d}(T)}{4}\left\{\left[F\left(R_{N}-\mathbf{1}\right) F^{\dagger}\right]_{\alpha \alpha}\left(1-\tanh \mu_{\alpha}\right)-\left[F^{*}\left(R_{\bar{N}}-\mathbf{1}\right) F^{T}\right]_{\alpha \alpha}\left(1+\tanh \mu_{\alpha}\right)\right\} .
\end{aligned}
$$

The equation for $R_{\bar{N}}$ can be found as explained in Sec. 3. We then obtain the coupled differential equations rather than the coupled integrodifferential equations. Let us recall here the origins of the communication terms in these equations. As for Eq. (33) the third term comes from $\delta \Gamma_{N}^{p}$ by the processes $(\mathrm{A})$ and $(\mathrm{B})$ and the fourth term comes from $\delta \Gamma_{N}^{d}$ by the process $(\mathrm{C})$. Similarly, $\delta \Gamma_{\nu}^{p}$ by $(\mathrm{A})$ and $(\mathrm{B})$ induces the third term and $\delta \Gamma_{\nu}^{d}$ by $(\mathrm{C})$ gives the fourth term in Eq. (34).

We can see that, although $R_{N}$ is introduced to be independent on the momentum $k$, the equation (33) does depend on $k$ through $\gamma_{N}^{d}(k)$ to which all the destruction and production rates of sterile neutrinos are proportional, and also through the effective Hamiltonian $H_{N}(k)$. Note that the rates of chemical potentials are independent on the momentum since they are proportional to $\gamma_{\nu}^{d}(T)$. In order to avoid this difficulty one might consider that the momentum in Eq. (33) is replaced as $k=k_{*} \sim T$, since such a mode is expected to give the dominant contribution to the number densities and also the asymmetries. However, we have to carefully choose $k_{*}$ to keep the conservation of the total lepton number. Under the considering situation, Eq. (22) is satisfied when

$$
0=\operatorname{tr}\left[\left.\frac{d R_{N}}{d t}\right|_{k=k_{*}}-\left.\frac{d R_{\bar{N}}}{d t}\right|_{k=k_{*}}+N_{D} \frac{d A}{d t}-N_{D} \frac{d A^{-1}}{d t}\right] .
$$

It can be shown that it is fulfilled if and only if $k_{*}=2 T$.

Interestingly, we observe that the rates with $k=2 T$ correspond to nothing but those obtained by the thermal average. To see this point, let us introduce the averaged $\gamma_{N}^{d}$ by

$$
\left\langle\gamma_{N}^{d}\right\rangle \equiv \frac{\int \frac{d^{3} k}{(2 \pi)^{3}} \rho^{\mathrm{eq}}(k) \gamma_{N}^{d}(k)}{\int \frac{d^{3} k}{(2 \pi)^{3}} \rho^{\mathrm{eq}}(k)}=\frac{N_{C} N_{D} h_{t}^{2} T}{128 \pi^{3}}
$$

and hence $\left\langle\gamma_{N}^{d}\right\rangle=\left.\gamma_{N}^{d}(k)\right|_{k=2 T}$. Note that $\gamma_{\nu}^{d}(T)=\left\langle\gamma_{N}^{d}\right\rangle$, which is accidentally obtained because of $N_{D}=2$. Therefore, the assumption (ii), i.e., the thermal average of the destruction rates in Eq. (6) , can then be achieved by the replacement $\gamma_{N}^{d}(k) \rightarrow\left\langle\gamma_{N}^{d}\right\rangle$. It should be noted that the 
averaged $\Gamma_{N}^{d \text { eq }}(k)$ in Eq. (11) is given by

$$
\left\langle\Gamma_{N}^{d \text { eq }}\right\rangle=3\left\langle\gamma_{N}^{d}\right\rangle\left[F^{\dagger} F\right]=\frac{9 h_{t}^{2}}{64 \pi^{3}} T\left[F^{\dagger} F\right]
$$

which is exactly the (total) destruction rate of sterile neutrinos introduced by Ref. [4] and used in Refs. [7, 11, 12.

In the end, by applying the assumptions (i) and (ii), our kinetic equations lead to the equation for $R_{N}$

$$
\begin{aligned}
\frac{d R_{N}}{d t}= & -i\left[\left\langle H_{N}\right\rangle, R_{N}\right]-\frac{3}{2}\left\langle\gamma_{N}^{d}\right\rangle\left\{F^{\dagger} F, R_{N}-\mathbf{1}\right\}+2\left\langle\gamma_{N}^{d}\right\rangle F^{\dagger}(A-\mathbf{1}) F \\
& -\frac{1}{2}\left\langle\gamma_{N}^{d}\right\rangle\left\{F^{\dagger}\left(A^{-1}-\mathbf{1}\right) F, R_{N}\right\} .
\end{aligned}
$$

We should compare the equations (38) and (34) with those in the previous works. Notice that they satisfy Eq. (35) and hence the lepton number is conserved for each mode with $k$.

In Ref. [4] the evolution of $R_{N}$ and $R_{\bar{N}}$ has been studied by using Eq. (38) where only the first two terms are taken into account and include no effect of chemical potentials. Ref. [7] has solved the coupled equations of $R_{N, \bar{N}}$ and chemical potentials (the equations for $\rho_{L, \bar{L}}$ in [7] are nothing but those of chemical potentials), where the third term in (38) and the first two terms in (34) are taken into account, however coefficients are different from the present ones. See the discussion below. The last terms in Eqs. (38) and (34) are original ones in the present paper.

We shall discuss in detail the differences from Ref. [11] where SU(2) degrees of freedom of lepton doublets are taken into account.\#3 As for the equation (38) there are two differences. One is the coefficient of the third term is "2" rather than " 3 ". The other one is the last term is missing in Ref. [11]. (Correspondingly, the equations for chemical potentials are also different.) We observe that the equations in Ref. [11] can be reproduced if one uses the destruction and production rates from the scattering process (A) or (B) (the rates for these two processes are the same as shown in Sec. 3), and multiplies the rates a factor of three to get the total rates. This is the assumption (iii) listed before.

As explained in Sec. 3, the processes (A) and (B) lead to the communication terms between $\rho_{N}$ and $\rho_{L}$, which induce the third term of Eq. (38). On the other hand, the process (C) connects between $\rho_{N}$ and $\rho_{\bar{L}}$ and gives the fourth term of Eq. (38). Note again that these communication terms arise as the corrections to the destruction and production rates of particles, not by hand. As a result, we can find the explicit form of such a term with a definite coefficient.

Having specified the differences between various sets of the kinetic equations to now, we can compare quantitatively the yields of the BAU. The results are summarized in Tab. 1, where we take the same parameter set described in Sec. 4, but both $M_{N}=10 \mathrm{GeV}$ and $100 \mathrm{MeV}$ are

\#3 The kinetic equations in [11 are reproduced from those in 17, 12, by replacing $\Gamma_{L} \rightarrow \Gamma_{L} / N_{D}$ and $\mu_{\alpha} \rightarrow$ $N_{D} \mu_{\alpha}$. The change of $\Gamma_{L}$ leads to the change of coefficient of the communication term in the equation of $\rho_{N}$ accordingly. The final value of the BAU in [11] decreases roughly by a factor of two due to these modifications. 


\begin{tabular}{|c||c|c||c|}
\hline Case & I & II & III \\
\hline \hline Kinetic eqs. & Ref. [1] & Eqs. (34) and (38) & Eqs. (12) and (20) \\
\hline Momentum dep. & Approx. (i), (ii), (iii) & Approx. (i), (ii) & Full \\
\hline Rates & $\Gamma_{N}^{d}=\left\langle 3 \Gamma^{d(\mathrm{~A})}\right\rangle$ & $\Gamma_{N}^{d}=\left\langle\Gamma^{d(\mathrm{~A}+\mathrm{B}+\mathrm{C})}\right\rangle$ & $\Gamma_{N}^{d}=\Gamma^{d, p(\mathrm{~A}+\mathrm{B}+\mathrm{C})}(k)$ \\
\hline$Y_{B}(10 \mathrm{GeV})$ & $3.8 \times 10^{-10}$ & $2.7 \times 10^{-10}$ & $2.9 \times 10^{-10}$ \\
$Y_{B}(100 \mathrm{MeV})$ & $5.8 \times 10^{-14}$ & $3.9 \times 10^{-14}$ & $5.4 \times 10^{-14}$ \\
\hline
\end{tabular}

Table 1: Comparison of $Y_{B}$ for cases I, II and III when $M_{N}=10 \mathrm{GeV}$ and $100 \mathrm{MeV}$. See the details in the text.

considered. The cases I and II correspond to Ref. [11] and to Eqs. (34) and (38), respectively. For comparison, we also show the results from Sec. 4 as the case III.

Let us first make the comparison between the cases I and II in which the momentum dependence in $\rho_{N}$ is approximately included. The momentum distributions of $\left[\rho_{N}(k)\right]_{22}$ and $\left[\Delta \rho_{N}(k)\right]_{\text {tot }}$ are shown in Fig. 8. Since the momentum dependence of $\rho_{N}$ is taken as (32), only the normalization is important. As seen in the occupation numbers, the yields of $N_{2}$ (and $N_{3}$ ) are almost the same because the production dominantly occurs through the second term in Eq. (38) which is common for both cases. On the other hand, the asymmetries are mainly generated through the communication terms of the kinetic equations, and then $Y_{B}$ can vary depending on the structure of these terms. We find that the BAU in the case II is smaller than I by $\sim 2 / 3$, and accordingly the distributions of $\left[\Delta \rho_{N}(k)\right]_{\text {tot }}$ are different by the same amount since $Y_{B} \propto Y_{\Delta N_{\text {tot }}}\left(=-Y_{\Delta L_{\text {tot }}}\right)$.

The origin of the factor $2 / 3$ can be understood as follows: Active flavor asymmetries $Y_{\Delta L_{\alpha}}$ are almost the same between two cases. This is because the three scattering processes equally contribute to the generation (as long as chemical potentials are small enough). On the other hand, only the processes (A) and (B) give a dominant contribution to $Y_{\Delta L_{\mathrm{tot}}}$ and $Y_{\Delta N_{\mathrm{tot}}}$ through the third term in Eq. (38). The contribution from the process (C) is suppressed by $\mathcal{O}\left(F^{2}\right)$. The difference in $Y_{B}$ is just coming from the change of the coefficient of this term. This is the impact of the new communication terms found in this analysis.

Finally, we turn to discuss the difference between the cases II and III in order to estimate the importance of the momentum dependence in $\rho_{N}$. Note that the difference lies only in the treatment of the momentum dependence between the two cases. When we take the smaller value of Majorana mass as $M_{N}=100 \mathrm{MeV}$ neutrino Yukawa coupling constants become sufficiently small that all the modes of interest can be away from the equilibrium one. As mentioned in Sec. 4, the momentum dependence in the rates boost (or disturb) the production of the occupation numbers and also the asymmetries for the modes with lower (or higher) momenta. These behaviors can be seen in the distributions in Fig. 8. In this case $Y_{B}$ is enhanced by about $40 \%$ due to the inclusion of the momentum dependence. 

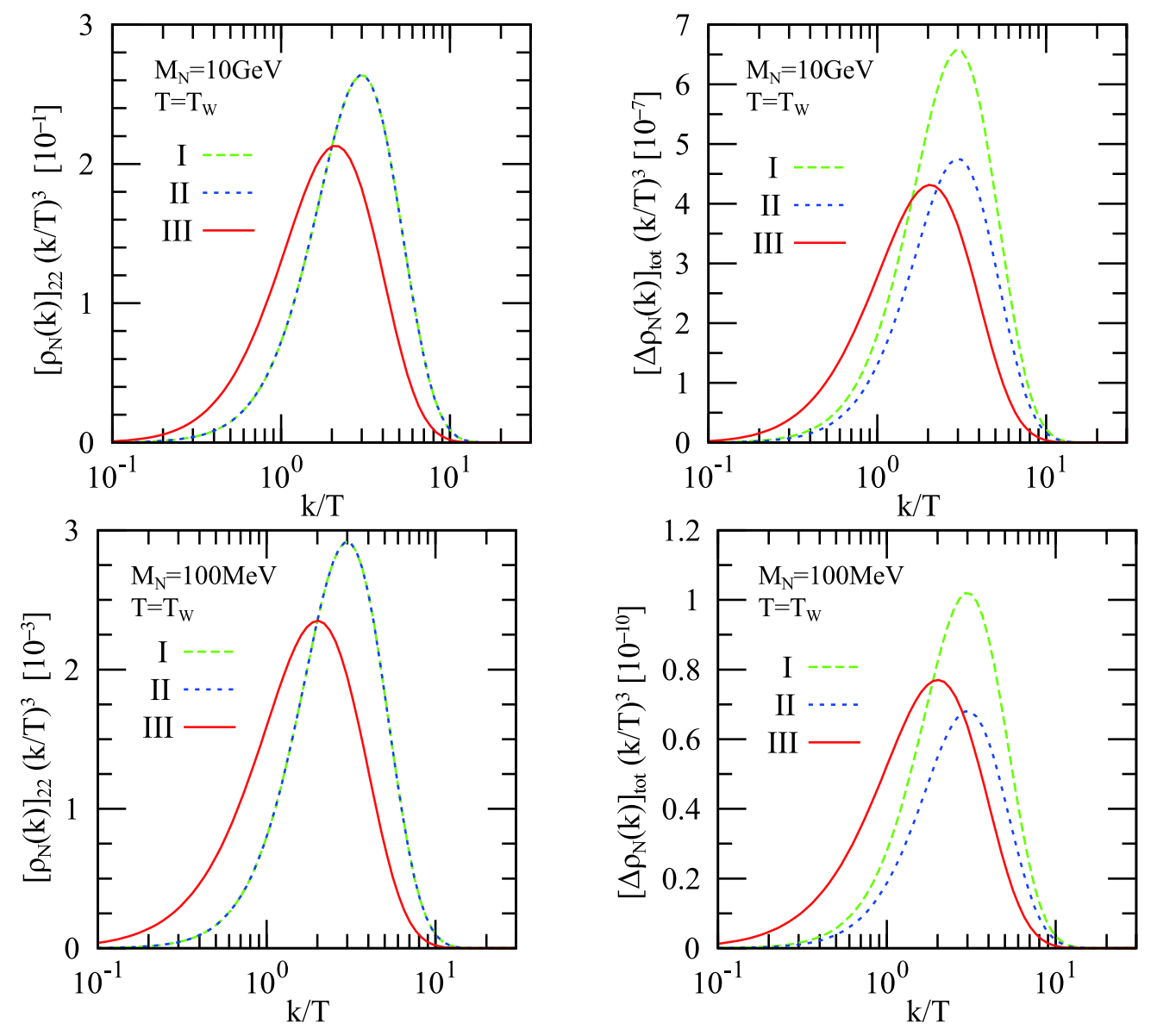

Figure 8: Comparison of momentum distributions of $\left[\rho_{N}(k)\right]_{22}$ (left panel) and $\left[\Delta \rho_{N}(k)\right]_{\text {tot }}$ (right panel) for the cases I, II and III in Tab. 1. The distributions are evaluated at $T=T_{W}$ and are multiplied by $(k / T)^{3}$. Here we take $M_{N}=10 \mathrm{GeV}$ and $100 \mathrm{MeV}$.

On the other hand, when $M_{N}=10 \mathrm{GeV}$, the large Yukawa coupling constants lead to another effect. The low momentum modes are likely to gets in thermal equilibrium and the matrix of densities becomes $\rho_{N} \rightarrow \rho^{\mathrm{eq}} \mathbf{1}$. The asymmetries carried by such modes receive the wash-out effect. This point is clearly seen by comparing the distributions $\left[\Delta \rho_{N}(k)\right]_{\text {tot }}$ of the cases II and III. As a result, the enhancement factor of $Y_{B}$ is reduced to about $10 \%$.

\section{Conclusions}

In this paper we have investigated baryogenesis induced by the flavor oscillation between sterile (right-handed) neutrinos $N_{2}$ and $N_{3}$ in the $\nu$ MSM. We have presented the kinetic equations for the matrices of densities of sterile neutrinos $\rho_{N}$ and $\rho_{\bar{N}}$ and chemical potentials of active (lefthanded) leptons $\mu_{\nu_{\alpha}}$ shown in Eqs. (12) and (20). By using these equations the time evolution for the matrices of densities can be traced for each mode, and the momentum distributions can be found at any moments relevant for baryogenesis. As a result they allow us to estimate the yield of the baryon asymmetry more precisely. 
We have evaluated the destruction and production rates of sterile and active leptons for the kinetic equations. Especially, we have paid a special attention to the following two respects. The first is to include the momentum dependence of the rate correctly, which is mandatory to obtain the equations of $\rho_{N, \bar{N}}$ for a given momentum. The second is to include the effect from the deviation of the initial or final state from the thermal equilibrium in the scattering process. As explained in Sec. 3, the second point is crucially important in the construction of the kinetic equations for baryogenesis in the $\nu$ MSM. We have shown that the terms connecting between sterile and active sectors, which are required for the successful baryogenesis, arise in a consistent way from the second effect to the rate.

To be concrete, the terms connecting the matrices of densities $\rho_{N}$ and $\rho_{L}$, which introduced in Ref. [7, appear as the corrections to the production rates $\delta \Gamma_{N}^{p}$ and $\delta \Gamma_{\nu}^{p}$ via the scattering processes (A) and (B). As shown in Sec. 5 the prefactor is different by $2 / 3$ to the previous result in Ref. [11]. Moreover, we have found the new terms which link $\rho_{N}$ to $\rho_{\bar{L}}$ (rather than $\left.\rho_{L}\right)$. They are induced as the corrections $\delta \Gamma_{N}^{d}$ and $\delta \Gamma_{\nu}^{d}$ to the destruction rates via the process (C). Importantly, such a term is proportional to the product of $\rho_{N, \bar{N}}$ and $\mu_{\nu_{\alpha}}$. Thus, our kinetic equations are no longer linear in $\rho_{N}, \rho_{\bar{N}}$ and $\mu_{\nu_{\alpha}}$. It should also be stressed that both types of these terms are required to ensure the conservation of the total lepton number. The impacts of these terms on the generation of the baryon asymmetry have been described in detail.

We have then investigated the numerical solutions of the kinetic equations. Because of the momentum dependence in the rates the production of sterile neutrinos is enhanced for the modes with $k / T \lesssim 1$ while diminished for the higher momentum modes. Consequently, the momentum distributions of $\rho_{N}$ and $\rho_{\bar{N}}$ are significantly distorted from the equilibrium one. This clearly shows the importance to utilize the momentum-dependent kinetic equations. It has also been found that the low modes get in the thermal equilibrium and the asymmetries carried by them receive the wash-out effect at higher temperatures. In our choice of the parameters, the mode with $k / T \simeq 2$ is the most significant for the occupation numbers as well as the asymmetries including the BAU.

The comparison with the previous works has also been discussed. We have first explained what kind of approximations are needed to reproduce the previous kinetic equations staring from the present ones. It is shown that the use of the destruction rate [4] obtained by the thermal average is crucial to ensure the lepton number conservation when we treat the momentum dependence of $\rho_{N}$ approximately. We have then compare quantitatively the yields of the BAU. Apart from the correct treatment of the momentum dependence, the present equations gives the smaller BAU by a factor of $\simeq 2 / 3$ compared with Ref. [11]. Within the specific choice of parameters, the inclusion of the momentum dependence enhances the BAU by about $40 \%$ and $10 \%$ for $M_{N}=100 \mathrm{MeV}$ and $10 \mathrm{GeV}$, respectively. This is because the enhancement in the generation of the asymmetry from the lower modes overcomes the suppression induced by the higher modes. On the other hand, such an amplification may be disturbed by the wash-out effect for large neutrino Yukawa coupling constants. 
The present analysis shows that the momentum distribution of the matrices of densities differs from the equilibrium one and the inclusion of their momentum dependence modifies the yield of the BAU in non-trivial manner depending on the parameters of neutrino Yukawa coupling constants and masses of sterile neutrinos. Therefore, it is important to explore the full parameter space of the $\nu \mathrm{MSM}$ accounting for the observed BAU by using the kinetic equations presented in this paper. This issue will be performed elsewhere.

\section{Acknowledgments}

We would like to thank E. Kh. Akhmedov, A. Kartavtsev and M. Shaposhnikov for valuable discussions and comments, and also to Particle and Astroparticle Division of Max-Planck-Institut für Kernphysik at Heidelberg for hospitality. The work of T.A. was supported by KAKENHI (No. 21540260) in JSPS. T.A. and S.E. are supported from Strategic Young Researcher Overseas Visits Program for Accelerating Brain Circulation in JSPS.

\section{References}

[1] A. Riotto and M. Trodden, Ann. Rev. Nucl. Part. Sci. 49 (1999) 35 arXiv:hep-ph/9901362.

[2] M. Fukugita and T. Yanagida, Phys. Lett. B 174 (1986) 45 .

[3] P. Minkowski, Phys. Lett. B 67 (1977) 421; T. Yanagida, in Proc. of the Workshop on the Unified Theory and the Baryon Number in the Universe, Tsukuba, Japan, Feb. 13-14, 1979, p. 95, eds. O. Sawada and S. Sugamoto, (KEK Report KEK-79-18, 1979, Tsukuba); Progr. Theor. Phys. 64 (1980) 1103 ; M. Gell-Mann, P. Ramond and R. Slansky, in Supergravity, eds. P. van Niewenhuizen and D. Z. Freedman (North Holland, Amsterdam 1980); P. Ramond, in Talk given at the Sanibel Symposium, Palm Coast, Fla., Feb. 25Mar. 2, 1979, preprint CALT-68-709 (retroprinted as hep-ph/9809459); S. L. Glashow, in Proc. of the Cargése Summer Institute on Quarks and Leptons, Cargése, July 9-29, 1979, eds. M. Lévy et. al, , (Plenum, 1980, New York), p707.

[4] E. K. Akhmedov, V. A. Rubakov and A. Y. Smirnov, Phys. Rev. Lett. 81 (1998) 1359 arXiv:hep-ph/9803255.

[5] V. A. Kuzmin, V. A. Rubakov and M. E. Shaposhnikov, Phys. Lett. B 155 (1985) 36.

[6] T. Asaka, S. Blanchet and M. Shaposhnikov, Phys. Lett. B 631 (2005) 151.

[7] T. Asaka and M. Shaposhnikov, Phys. Lett. B 620 (2005) 17. 
[8] A. Boyarsky, O. Ruchayskiy and M. Shaposhnikov, Ann. Rev. Nucl. Part. Sci. 59 (2009) 191 [arXiv:0901.0011 [hep-ph]].

[9] F. L. Bezrukov and M. Shaposhnikov, Phys. Lett. B 659 (2008) 703 arXiv:0710.3755 [hep-th]].

[10] M. Shaposhnikov, JHEP 0808 (2008) 008 [arXiv:0804.4542 [hep-ph]].

[11] T. Asaka and H. Ishida, Phys. Lett. B 692 (2010) 105 [arXiv:1004.5491 [hep-ph]].

[12] L. Canetti and M. Shaposhnikov, JCAP 1009 (2010) 001 arXiv:1006.0133 [hep-ph]].

[13] A. D. Dolgov, Sov. J. Nucl. Phys. 33 (1981) 700 [Yad. Fiz. 33 (1981) 1309].

[14] R. Barbieri and A. Dolgov, Nucl. Phys. B 349 (1991) 743.

[15] G. Sigl and G. Raffelt, Nucl. Phys. B 406 (1993) 423.

[16] A. D. Sakharov, Pisma Zh. Eksp. Teor. Fiz. 5 (1967) 32 [JETP Lett. 5 (1967 SOPUA,34,392-393.1991 UFNAA,161,61-64.1991) 24].

[17] B. Dasgupta, A. Dighe, G. G. Raffelt and A. Y. Smirnov, Phys. Rev. Lett. 103 (2009) 051105 arXiv:0904.3542 [hep-ph]].

[18] H. Duan, G. M. Fuller, Y. -Z. Qian, Ann. Rev. Nucl. Part. Sci. 60 (2010) 569-594. arXiv:1001.2799 [hep-ph]].

[19] H. A. Weldon, Phys. Rev. D 26 (1982) 2789.

[20] G. L. Fogli, E. Lisi, A. Marrone, A. Palazzo and A. M. Rotunno, Phys. Rev. D 84 (2011) 053007 arXiv:1106.6028 [hep-ph]].

[21] Y. Burnier, M. Laine and M. Shaposhnikov, JCAP 0602 (2006) 007 [hep-ph/0511246].

[22] T. Asaka, S. Eijima and H. Ishida, JHEP 1104 (2011) 011 [arXiv:1101.1382 [hep-ph]]. 